\title{
Commentary on Ruiz-Arguelles editorial for Medicina Universitaria
}

\author{
Robert Peter Gale* \\ Department of Immunology and Inflammation, Centre for Hematology, Imperial College London, London, United Kingdom
}

Be not the first by whom the new is tried. Nor yet the last to lay the old aside.

Alexander Pope (A Essay on Criticism. 1711)

This issue of Medicina Universitaria contains an editorial from Prof. Ruiz-Arguelles discussing several important and controversial topics. The fundamental issue is whether everyone with cancer needs and/or benefits from the newest drugs and whether physicians are complicit in promoting these new drugs. Ruiz-Arguelles' focus is on two cancers, plasma cell myeloma (PCM) and chronic lymphocytic leukemia (CLL). The question is especially important in developing countries where these new drugs may not yet, or ever, be approved, available and/or affordable.

Since 2010, the US Food and Drug Administration (FDA) has approved six new drugs for PCM including carfilzomib, pomalidomide, daratumumab, ixazomib, elotuzumab, and panobinostat and seven approvals for CLL including ofatumumab, ibrutinib, duvelisib, idelalisib, obinutuzumab, venetoclax, and acalabrutinib. The question is how good are these drugs and do people with PCM or CLL really need them to have reasonable outcomes?

First, have these drugs improved outcomes? Yes and no. Many were approved based on a surrogate endpoint such as complete or overall response rate, response duration, and/or progression-free survival (PFS) rather than survival. For example, between 2009 and 2014, the US FDA approved 83 cancer drugs, 55 based on surrogate outcomes including 31 based on overall response rate and 24 based on PFS ${ }^{1}$. All accelerated approvals and one-half of conventional approvals were based on treatment effects with surrogate outcomes. In a recent review, fewer than one-half of drugs approved based on a PFS endpoint were shown to improve survival or quality of life $(\mathrm{QoL})^{2}$. Similarly, between 2009 and 2013, the European Medicines Agency approved 48 cancer drugs only one-half of which were proved to increase survival or improve QoL ${ }^{3}$.

Another problem with several new drug approvals is the choice of an inappropriate comparator therapy in phase-3 trials. For example, several recent PCM drugs were approved based on a dexamethasone - only comparator arm and several CLL drugs based on a chlorambucil and prednisone comparator arm.

A third issue is the use of a hazard ratio to prove benefit. A new drug compared with an old drug might have a hazard ratio for PFS of 0.75 with $95 \%$ confidence interval of 0.51-0.99 typically written as $H R=0.75(0.51,0.99)$ with $p=0.04$. Elsewhere I describe confusion of what $p$-value is and what it means ${ }^{4,5}$. However, simply put this implies the risk of progression or death could be halved. However, it also means that it could be decreased by only $1 \%$ or anywhere in between these values, though most likely $75 \%$. Although we cannot convert directly from a hazard ratio to median survival this hazard ratio is compatible with a very modest increase in median 
survival, say from 6 to 7.5 months, a 1.5 month (45 days) PFS benefit. The American Society of Clinical Oncology (ASCO) recommends approval of drugs increasing survival by 3 months $^{6}$. Although this magnitude of benefit might be meaningful in advanced lung and pancreas cancers, it is a low threshold in persons with PCM and CLL. Elsewhere Barosi and I discuss this issue in the context of PCM, Monserrat and I in the context of CLL and Estey et al. and I in the context of acute myeloid leukemia ${ }^{7-9}$.

Finally, there is the issue of new drugs approved based on clinical trials claiming non-inferiority rather than superiority to an old drug ${ }^{10}$. Although this is sometimes appropriate, for example, when a conventional drug has an adverse risk profile, this is rarely the basis for using a new, more expensive drug.

Ruiz-Arguelles suggests that one reason many new drugs gain favor with physicians and patients is because they are promoted by nationally or internationally by disease experts often referred to as key opinion leaders (KOLs) and drug companies, often, he suggests, in cahoots. True. However, there are several complex considerations here. KOLs are frequently principle investigators in the clinical trial(s) resulting in a new drug approval. Often, they believe, sometimes strongly or even too strongly, the new drug is an important advance. In psychology, this process is us known as confirmation bias. Having agreed to study a new drug, investing several years, often with considerable effort and problems working with ethical committees, clinical research organizations ${ }^{11}$; sometimes known as clinical research aggravations, drug company study managers, etc., one wants to believe their effort worthwhile. Otherwise, you would seem to have wasted your time, energy, and perhaps destroyed your marriage, missed your children growing up, both or perhaps something even worse (a fling in Ixtapa?).

However, we need to acknowledge other considerations such as the requirement to publish for academic promotion and advancement. It's far easier to publish a study claiming a new (typically more expensive) drug is better than an old (typically cheaper) drug than the converse. Moreover, there are also some less altruistic considerations. Everyone likes to address a large, admiring audience, have your headshot (typically of you 20 years ago) projected on a huge screen, travel abroad, be feted at a reception and dine in La Nacional in Monterrey or El Mural de los Poblanos in Puebla. This is human nature and we cannot unfairly fault them. Generic drug makers are unlikely to take you and the meeting organizers to Pujol in Ciudad de Mexico for dinner. Everyone likes to be liked.

For balance, let's consider a world where drug companies do not develop new drugs for PCM, CLL and other cancers. First, we would be left with melphalan, chlorambucil, dexamethasone, and prednisone. Not a happy situation. Almost all new cancer drugs in the past 30 years have come from drug companies, not academia. However, there are other far-reaching implications. For example, annual educational meetings such as those of the American Society of Hematology (ASH) and the European Hematology Association (EHA) would have 1500 rather than 15,000 attendees. About one-third to one-half of the attendees are drug company employees or physicians sponsored to attend by the meeting by drug companies. Much of these annual meetings funding comes from the drug industry rather than registration fees. ASH and ASCO charge drug companies substantial amounts for exhibition space and for the opportunity to present their new drugs in so-called product theatres. Imagine a world with no free cappuccino or frozen yogurt, video games, and giveaways. Blood and the journal of clinical oncology are filled with advertisements for new drugs. I doubt these journals would be able to achieve their widespread distributions at a reasonable cost to society members without this advertising income. Likewise their websites where you are bombarded by unsolicited advertisements for new drugs. And think of the exhibit areas of $\mathrm{ASH}, \mathrm{EHA}$, or the ASCO annual meetings. My point is that although it is popular to disparage the drug industry for promoting new drugs with sometimes marginal benefits and high costs, the medical community benefits greatly from the investment of these drug companies. Much of our medical education in diverse areas is indirectly supported by the drug industry. Our lives would be entirely different if drug companies developing and promoting new drugs stopped. And we would be eating tacos at a roadside stand (which can be excellent) rather than black cod in miso at Nobu. Remember, the estimated cost of bringing a new cancer drug to approval is about $\$ 700$ million to 2 billion US $\$ 12$. This money must come from somewhere and be returned with a profit to stockholders.

Next, a comment on the nomenclature surrounding new drugs. PCM and CLL experts like to refer to any drug developed after melphalan and chlorambucil as novel. Thalidomide was approved by the US FDA in 1998, 21 years ago. It was not new and original, not like anything seen before if we consider its approval for morning sickness associated with pregnancy in Germany in 
1956, 42 years earlier. Admittedly, daratumumab, approved by the US FDA last year, is new and original as is ibrutinib and acalabrutinib, ofatumumab, etc. My point is that there comes a time to stop calling every post-melphalan and -chlorambucil drug novel. KOLs and drug companies seem to me equally responsible for this misplaced enthusiasm but equally reluctant to change. For example, who wants a drug which is old and tired versus a drug which is new and novel. Not me. Its like asking whether its better to be young, beautiful and it's rich or old, ugly, and poor.

My bottom line is some of these new drugs are sometimes important scientific advances such as daratumumab in PCM and ibrutinib and venetoclax in CLL. Patients and physicians like the idea of not needing to receive chemotherapy. However, we should recall the chemotherapy means using drugs (rather than radiation surgery) to treat a disease. Ibrutinib and venetoclax are also chemotherapy, maybe even daratumumab. However, the impact of these new drugs on PFS and survival is mostly modest and not everyone needs them. We are not (yet) curing people with PCM or CLL but trying to help them live longer and better quality lives. In an environment of limited resources with needs to reduce infant mortality, childhood vaccination programs, malaria prevention, etc., new drugs are unlikely to be the most effective investment of health-care resources in developing countries.
Despite the above comments, criticisms, and admonitions, I am standing by to renounce all of these noble if a drug company wants to sponsor me to lecture on novel goals for PCM or CLL in Cuidad de Mexico with dinner at Quintonil thrown in.

\section{References}

1. Kemp R, Prasad V. Surrogate endpoints in oncology: when are they acceptable for regulatory and clinical decisions, and are they currently overused? BMC Med. 2017:15:134.

2. Kim C, Prasad V. Cancer drugs approved on the basis of a surrogate end point and subsequent overall survival: an analysis of 5 years of US food and drug administration approvals. JAMA Intern Med. 2015;175:1992-4

3. Davis C, Naci H, Gurpinar E, Poplavska E, Pinto A, Aggarwal A. Availability of evidence of benefits on overall survival and quality of life of cancer drugs approved by European medicines agency: retrospective cohort study of drug approvals 2009-13. BMJ. 2017;359:j4530.

4. Gale RP, Zhang MJ. What is the P-value anyway? Bone Marrow Transplant. 2016;51:1439-40.

5. Gale RP, Hochhaus A, Zhang MJ. What is the (p-) value of the P-value? Leukemia. 2016;30:1965-7.

6. Ellis LM, Bernstein DS, Voest EE, Berlin JD, Sargent D, Cortazar P, et al. American society of clinical oncology perspective: raising the ba for clinical trials by defining clinically meaningful outcomes. J Clin Oncol. 2014;32:1277-80

7. Barosi G, Gale RP. Is lenalidomide the standard-of-care after an autotransplant for plasma cell myeloma? Leukemia. 2019;33:588-96.

8. Montserrat E, Gale RP. Predicting the outcome of patients with chronic lymphocytic leukemia: progress and uncertainty. Cancer. 2019;125:3699-705.

9. Estey E, Karp JE, Emadi A, Othus M, Gale RP. Recent drug approvals for newly diagnosed acute myeloid leukemia: gifts or a Trojan horse? Leukemia. 2020. doi: https://doi.org/10.1038/s41375-019-0704-5.

10. Non-Inferiority Clinical Trials to Establish Effectiveness; 2016. Available from: https://www.fda.gov/media/78504/download.

11. Sekeres M. Contract Research Agonizations. ASH Clinical News; 2017. Available from: https://www.ashclinicalnews.org/viewpoints/editors-corner/contract-research-agonizations.

12. Prasad V, Mailankody S. Research and development spending to bring a single cancer drug to market and revenues after approval. JAMA Intern Med. 2017;177:1569-75. 